\title{
Desarrollo de una herramienta de aprendizaje experiencial en el área de Seguridad y Salud en el Trabajo soportada en Realidad Virtual
}

Development of an experiential learning tool in the area of Occupational Safety and Health supported by Virtual Reality

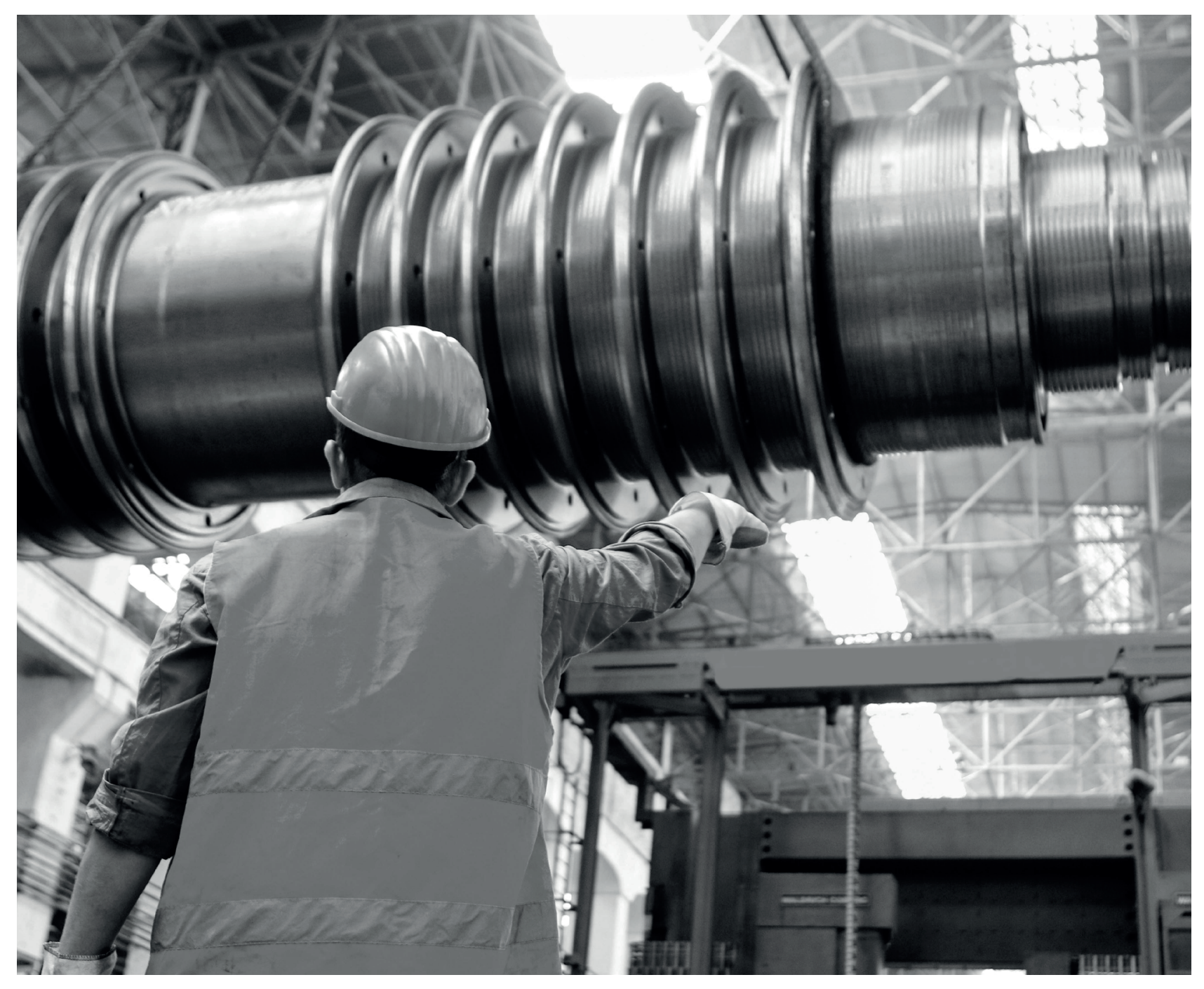




\title{
Desarrollo de una herramienta de aprendizaje experiencial en el área de Seguridad y Salud en el Trabajo soportada en Realidad Virtual ${ }^{1}$
}

\author{
Development of an experiential learning tool in the area of \\ Occupational Safety and Health supported by Virtual Reality
}

\section{Mariana Acuña-Rangel ${ }^{2}$, Juan Camilo Lésmez-Peralta ${ }^{3}$, Maira Camila Paba-Medina4, Luis Miguel Salas-Chia ${ }^{5}$}

Artículo recibido en enero 19 de 2021; artículo aceptado en abril 07 de 2021

\begin{abstract}
Este artículo puede compartirse bajo la Licencia Creative Commons Atribución-NoComercial-Compartirlgual 4.0 Internacional y se referencia usando el siguiente formato: Acuña-Rangel, M., Lésmez-Peralta, J. C., Paba-Medina, M. C. y Salas-Chia, L. M. (año). Desarrollo de una herramienta de aprendizaje experiencial en el área de Seguridad y Salud en el Trabajo soportada en Realidad Virtual. I+D Revista de

Investigaciones, 16(2), 150-157. https://doi.org/10.33304/revinv.v16n2-2021014
\end{abstract}

\section{Resumen}

La Seguridad y Salud en el Trabajo es una disciplina cuya implementación en las organizaciones ha cobrado cada vez más importancia en los últimos años. Por esta razón, nace la necesidad de encontrar herramientas que soporten el proceso de enseñanza y la transmisión de conocimientos para la vida profesional. Partiendo de la metodología Design Thinking, se genera el proceso de ideación y creación de una herramienta gamificada de aprendizaje experiencial soportada en Realidad Virtual. Como resultado, se obtuvieron cuatro escenarios que simulan la operación de los trabajadores en diferentes sectores productivos. La validación de la herramienta se hizo mediante un instrumento de evaluación de cinco factores, diligenciado por un grupo de prueba que interactuó con el programa. Finalmente, la herramienta presentó aval por parte de los usuarios manifestando que esta es una experiencia educativa que les facilita aprender los conceptos colocándolos en práctica en un ambiente simulado.

Palabras clave: Aprendizaje experiencial, Design thinking, herramienta educativa, realidad virtual, seguridad y salud en el trabajo.

\footnotetext{
${ }^{1}$ Artículo de investigación, de enfoque cualitativo, resultado de un proyecto de investigación de la convocatoria interna de la Universidad Industrial de Santander, InnovaTIC 2020, perteneciente al área de Ciencias sociales y gestión organizacional, subárea de seguridad y salud en el trabajo, desarrollado en el Grupo de Investigación Finance \& Management, fue financiado por la Universidad Industrial de Santander (Bucaramanga, Colombia). Dirección: Carrera 27, Calle 9, Ciudad Universitaria, PBX: (60) (7) 6344000. Fecha de inicio: 27 de agosto de 2020. Fecha de terminación: 31 de diciembre de 2020.

${ }^{2}$ Ingeniera Industrial, Universidad Industrial de Santander. Vinculada al Grupo de Investigación Finance \& Management, Universidad Industrial de Santander (Bucaramanga, Colombia). Dirección: Carrera 27, Calle 9 Ciudad Universitaria, PBX: (60) (7) 6344000. ORCID ID: https://orcid.org/00000001-9895-6809. Correo electrónico institucional: MARIANA.ACUNA@correo.uis.edu.co.

${ }^{3}$ Magíster en Gerencia de Negocios - MBA, Universidad Industrial de Santander. Vinculado al Grupo de Investigación Finance \& Management, Universidad Industrial de Santander (Bucaramanga, Colombia) Dirección: Carrera 27, Calle 9, Ciudad Universitaria, PBX: (60) (7) 6344000. ORCID ID: https://orcid.org/0000-0003-0400-0270. Correo electrónico institucional: jclesper@uis.edu.co.

${ }^{4}$ Ingeniera Industrial, Universidad Industrial de Santander. Vinculada al Grupo de Investigación Finance \& Management, Universidad Industrial de Santander (Bucaramanga, Colombia) Dirección: Carrera 27, Calle 9 Ciudad Universitaria, PBX: (60) (7) 6344000. ORCID ID: https://orcid.org/00000002-0453-9367. Correo electrónico institucional: maira.paba@correo.uis.edu.co.

${ }^{5}$ Ingeniero de Petróleos, Universidad Industrial de Santander. Vinculado al Grupo de Investigación Finance \& Management, Universidad Industrial de Santander (Bucaramanga, Colombia). Dirección: Carrera 27, Calle 9 Ciudad Universitaria, Edificio Escuela de Estudios Industriales y Empresariales - EEIE, Oficina 410, PBX: (60) (7) 6344000, Ext. 1425. ORCID ID: https://orcid.org/0000-0001-9041-9291. Correo electrónico institucional: luis.salas@ correo.uis.edu.co.
} 
Mariana Acuña Rangel, Juan Camilo Lésmez Peralta, Maira Camila Paba Medina, Luis Miguel Salas Chía

Desarrollo de una herramienta de aprendizaje experiencial en el área de Seguridad y Salud en el Trabajo soportada en Realidad Virtual

\begin{abstract}
Occupational Health and Safety is a discipline whose implementation in organizations has become increasingly important in the recent years. For this reason, there is a need to find tools that support the teaching process and the transmission of knowledge to a professional life. Based on the Design Thinking methodology, the process of ideation and creation of a gamified experiential learning tool supported by Virtual Reality is generated. As a result, four scenarios were obtained that simulate the operation of workers in different productive sectors. The validation of the tool was done by means of a five-factor evaluation instrument, filled out by a test group that interacted with the program. The outcome of this study turned out positive since, the tool was endorsed by the users, who stated that this is an educational experience that makes it easier for them to learn the concepts by putting them into practice in a simulated environment.
\end{abstract}

Keywords: Experiential learning, design thinking, educational tool, virtual reality, occupational safety and health.

\section{Introducción}

El avance en los procesos empresariales, así como las normativas internacionales que velan por el bienestar de sus empleados, han permitido que las actividades desarrolladas en las industrias refuercen su enfoque en la Seguridad y Salud en el Trabajo (SST). La SST debe ser aplicada en las actividades de cualquier nivel de las empresas con el objetivo de garantizar un trabajo sin riesgos y sin efectos colaterales ocasionados por los accidentes 0 enfermedades laborales (Ministerio del Trabajo, 2020). El conocimiento de estos sistemas por parte de todos los trabajadores, desde los operarios hasta los administrativos, es de gran importancia ya que este tipo de riesgos se encuentran latentes en cualquier puesto de trabajo (Hernández Palma et al., 2017).

Por esta razón, la necesidad de contar con elementos que permitan la difusión y entrenamiento en SST en las aulas de clase ha generado la oportunidad de realizar una herramienta soportada en Realidad Virtual (RV) como componente de apoyo para una educación experiencial en esta materia. Este tipo de aprendizaje se convierte en una filosofía para mejorar los procesos de aprendizaje en personas adultas, el cual tiene como punto de partida el principio de que las personas aprenden mejor cuando entran en contacto directo con sus propias experiencias (Hernández Palma et al., 2017).

En esta medida, la RV se reconoce como un instrumento en el que la necesidad de desplazarse hasta un sitio de trabajo deja de ser un requisito, permitiendo así la inclusividad de la herramienta a aquellas personas con limitaciones motrices las cuales no pueden acceder a ciertas locaciones para su estudio (Castaño Calle et al., 2019), sin dejar de lado las oportunidades y limitaciones de acceso a la tecnología por parte de diferentes sectores de la población de este país (Cruz Carbonell et al., 2020). El desarrollo de la herramienta se basó en la metodología de Design Thinking, la cual, para el caso particular de este estudio, sus cinco pasos se agruparon en tres pasos: (1) selección del enfoque (empatizar y definir), (2) proceso de ideación (idear) y (3) creación del prototipo y validación (prototipar y evaluar). En este sentido, cada uno de estos pasos está integrado por una o más fases de las cinco originales del Design Thinking. La agrupación de fases se realizó debido a que el tiempo de ejecución era reducido, por tanto, los investigadores decidieron fusionar fases para optimizar el proceso. La herramienta fue evaluada y validada en un ambiente controlado a través de una encuesta que tuvo en cuenta diversos factores que evaluaron su pertinencia y correcto funcionamiento, arrojando un nivel de aprobación adecuado de esta.

\section{Realidad Virtual y aprendizaje experiencial}

En el actual contexto, que se caracteriza por tener una sociedad rápidamente cambiante, los jóvenes se enfrentan a múltiples retos que les exigen responder con agilidad ante las necesidades del entorno. Así, una de las competencias más demandadas está relacionada con la habilidad de aprender de forma autónoma a través de hábitos de lectura y estudio individual (Moreno Mendoza y Coronel Arenas, 2018; Romero Ariza, 2010) y adquirir los conocimientos y habilidades de manera adecuada, de tal manera que el estudiante pueda retener estos conceptos a lo largo de su vida.

Desde esta perspectiva surge el aprendizaje experiencial, el cual, promueve la capacidad de aprender a aprender y conectar situaciones reales de la vida con los conceptos y conocimientos adquiridos, por lo que se fomenta la capacidad de aplicar lo aprendido (Romero Ariza, 2010). Smith (2010) señala que uno de los rasgos característicos del aprendizaje experiencial es que involucra al individuo en una interacción directa con aquello que se está estudiando. Lo anterior es positivo en la medida que esta metodología puede ser considerada como la forma más primitiva de aprender, pues permite tener un contacto directo con los elementos implicados en la aplicación real de los conocimientos y habilidades. No obstante, de acuerdo con Bhat (2010), el aprendizaje experiencial no se limita a la práctica, sino que también incluye procesos cognitivos de aprendizaje y afirma la importancia de la reflexión crítica en la formación académica. 
Mariana Acuña Rangel, Juan Camilo Lésmez Peralta, Maira Camila Paba Medina, Luis Miguel Salas Chía Desarrollo de una herramienta de aprendizaje experiencial en el área de Seguridad y Salud en el Trabajo soportada en Realidad Virtual

Como una aplicación del aprendizaje experiencial surge la Realidad Virtual, una simulación interactiva y tridimensional (3D) diseñada con el objetivo de permitir al usuario no solo ver, sino también manipular los contenidos del ambiente simulado (Escartín, 2000). La Realidad Virtual ha sido reconocida como una tecnología de gran impacto en la educación porque hace posible que los alumnos interactúen en escenarios simulados que en la vida real podrían ocurrir como un evento fortuito o representar un peligro, tal como un incendio o una catástrofe natural. Así, las posibilidades que ofrece la RV permiten a los estudiantes tener un aprendizaje experiencial, en la medida que aprenden haciendo y mejorar así sus habilidades prácticas.

\section{Seguridad y Salud en el Trabajo}

La Seguridad y Salud en el Trabajo (SST) es una temática de vital importancia en todos los sectores laborales, pues garantiza la protección de la vida misma, lo que evita accidentes y enfermedades laborales y trabaja por la concientización de los empleados y estudiantes sobre los riesgos laborales, así como sobre la posibilidad de evitarlos (Burgos García, 2010). Por esta razón, el concepto de la SST está inmerso en las necesidades empresariales, comunitarias y globales, por lo que ha instado a los gobiernos nacionales e internacionales a plantear políticas públicas en esta materia (Álvarez Torres y Riaño Casallas, 2018).

Las preocupaciones alrededor de esta temática han surgido desde hace algunos años a causa de las elevadas cifras de muertes y accidentes ocasionadas por el trabajo; en 1959, con la Resolución 112 sobre servicios de Medicina del Trabajo se acuña el término "salud ocupacional", orientado a los objetivos de: (I) Mantenimiento y promoción de la salud y capacidad de trabajo de los empleados, (II) Mejoramiento de los ambientes laborales, y (III) Desarrollo de culturas de trabajo (Eijkemans, 2003, p. 1).

No obstante, la Organización Internacional del Trabajo (OIT) ha difundido la noción de Seguridad y Salud en el Trabajo entendida como:

Una disciplina que trata de la prevención de las lesiones y enfermedades relacionadas con el trabajo y de la protección y promoción de la salud de los trabajadores. La salud en el trabajo conlleva a la promoción y el mantenimiento del más alto grado de salud física y mental y de bienestar de los trabajadores en todas las ocupaciones (Organización Internacional del Trabajo, 2011, p. 1).

\section{Metodología}

Una metodología de ideación y creación de herramientas que ha tomado fuerza en los últimos años para facilitar la innovación en diferentes escenarios es el Design Thinking
(Castillo-Vergara et al., 2014), la cual se usa como punto de partida para el diseño y desarrollo la perspectiva de los usuarios finales; de esta forma, se pueden detectar problemas y necesidades, así como ofrecer soluciones efectivas para cada una de ellas (Brown, 2008). El Design Thinking se define como un proceso tanto analítico como creativo, que invita a los participantes a hacer parte de ambientes que facilitan oportunidades para la generación de ideas innovadoras (Razzouk y Shute, 2012). Algunos autores consideran que el Design Thinking es tanto un proceso como una herramienta cognitiva, la cual permite resolver problemas en formas descritas como "originales" e "inesperadas" (Dell'Era et al., 2020).

Partiendo de la metodología propuesta por IDEO (2014), se describen a continuación las etapas utilizadas para el diseño y desarrollo de la herramienta de aprendizaje experiencial significativo apoyada en Realidad Virtual.

\section{Selección del enfoque}

Para esta etapa, el diseño de la herramienta inicia con los primeros pasos de la metodología de Design Thinking: empatizar y definir. La fase de empatizar tiene un enfoque humanístico de la población objetivo, donde se pueden conocer sus necesidades y problemáticas a través del análisis de su situación actual, en el que se busca que los investigadores adquieran conocimientos sobre los usuarios que harán uso del producto final (Arias-Flores et al., 2019). En esta fase, se empleó una herramienta que permite identificar a la población objetivo denominada mapa de empatía, usada en esta ocasión por el equipo de diseño, conformado por seis personas, para dar respuestas a preguntas como: ¿Quién es el usuario con el que vamos a empatizar? ¿Qué necesita hacer de una forma diferente? ¿Qué piensa y siente el usuario? ¿Qué ve? ¿Qué dice? ¿Qué oye? ¿Cómo actúa?, cuestionamientos a través de los cuales se identificó a la población que será impactada con el uso de la herramienta, correspondiente a estudiantes de pregrado que cursan la asignatura de Seguridad y Salud en el Trabajo, entendiendo así su situación actual, sus estímulos y su comportamiento frente a los mismos (Villaescusa Alejo, 2019).

En la fase de Definir se organizó la información obtenida sobre los alumnos estudiados en la etapa anterior, identificando sus características más relevantes y permitiendo establecer el foco de acción en el que se concentrará la herramienta educativa partiendo de las necesidades de los mismos (López Jiménez et al., 2020). Al finalizar esta etapa se pudo conocer quién es el usuario, cuál es su necesidad y hacia dónde se enfocará la herramienta de aprendizaje experiencial significativo apoyada en Realidad Virtual.

\section{Proceso de ideación}

Posterior a la selección del enfoque, el equipo inicia el proceso de ideación partiendo de la fase Idear del 
Mariana Acuña Rangel, Juan Camilo Lésmez Peralta, Maira Camila Paba Medina, Luis Miguel Salas Chía

Desarrollo de una herramienta de aprendizaje experiencial en el área de Seguridad y Salud en el Trabajo soportada en Realidad Virtual

Design Thinking, la cual busca que se genere la mayor cantidad de ideas posibles en relación con el problema previamente identificado (Castillo-Vergara et al., 2014). Una de las sugerencias propias de esta etapa es hacer uso de un equipo multidisciplinario que, mediante trabajo colaborativo, genere soluciones creativas frente a la problemática identificada en la población objetivo, tomando ventaja de la diversidad de conocimientos, habilidades y personalidades del grupo (Arias-Flores et al., 2019).

La herramienta utilizada por los investigadores para esta etapa fue el brainstorming, o lluvia de ideas, en la que un grupo de cinco individuos generó un gran número de ideas posibles dentro de un periodo de 20 minutos, siendo esta una herramienta que facilitó la generación de múltiples intervenciones voluntarias, aumentando además la creatividad en los participantes del ejercicio (De la Herrán, 2011). Cuando se finalizó esta etapa se definieron los componentes de la herramienta, así como la interacción de los usuarios con la misma.

\section{Creación del prototipo y validación}

La cuarta fase en el Design Thinking se denomina Prototipar, en la que se desarrolló un modelo tangible de la idea definida en la etapa anterior el cual contiene todos los componentes, mecánicas y dinámicas planteadas (Castaño García y González Morales, 2020).

Finalmente, luego de tener el prototipo físico de la herramienta, se procedió a realizar la fase final de la metodología Design Thinking: Evaluar. En esta, se probó el prototipo en un ambiente controlado (Arias-Flores et al., 2019) y se registraron las reacciones y opiniones de los participantes mediante un instrumento que midió factores relacionados con la usabilidad de la herramienta. De forma concreta, se utilizó una encuesta formulada por el equipo ejecutor del proyecto, validada y aplicada inicialmente con 15 estudiantes.

Al concluir esta etapa se obtuvo la versión final de la herramienta de aprendizaje experiencial significativo apoyada en Realidad Virtual.

\section{Resultados}

Con base en el proceso metodológico propuesto se obtuvieron resultados por cada una de estas fases como se expone a continuación.

Durante la selección del enfoque mediante los mapas de empatía empleados, se identificó que el usuario necesitaba una herramienta que le permitiera tener acceso a la educación relacionada a la seguridad y salud en el trabajo de una manera más práctica y experimental, permitiéndole interactuar en escenarios que simulen la realidad, pero donde se elimine la dificultad para acceder a estos.
En la etapa de ideación, mediante el método de lluvia de ideas, los participantes plantearon que la herramienta debía contar con cuatro escenarios, cada uno de los cuales representa el día a día en tres empresas pertenecientes a diferentes sectores productivos: una fábrica de muebles, una metalmecánica y una fábrica de textiles, escenarios dentro de los cuales se encuentra la representación de una serie de peligros a los que el usuario podrá enfrentarse para identificar y clasificar los riesgos a los que están expuestos los trabajadores.

En el proceso de creación del prototipo se desarrolló la herramienta con una interfaz que toma como punto de partida la captura de imágenes $360^{\circ}$ de escenarios reales enlazados a Realidad Virtual mediante el uso de un smartphone acoplado a unas gafas especializadas para experimentar esta tecnología (Ver Figuras 1, 2, 3, 4 y 5).

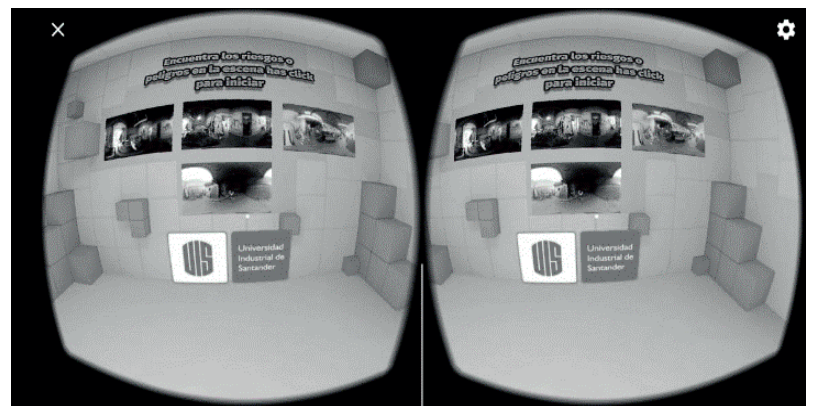

Figura 1. Pantalla inicial donde el usuario puede seleccionar en cuál de los cuatro escenarios disponibles va a interactuar. Fuente: autores a partir de la aplicación desarrollada.

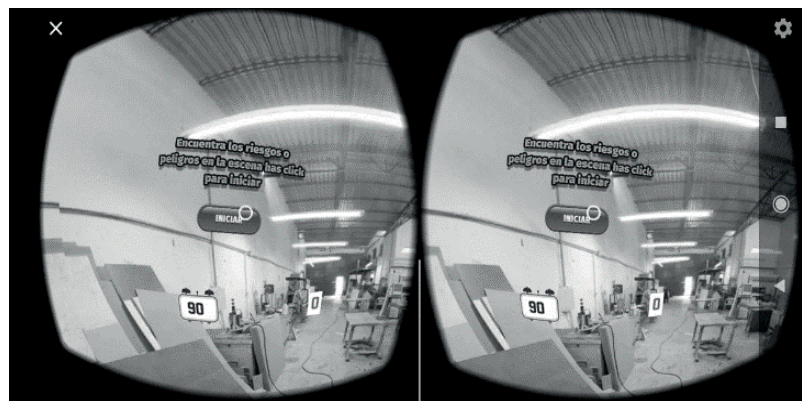

Figura 2. Captura de uno de los escenarios planteados. Fuente: autores a partir de la aplicación desarrollada.

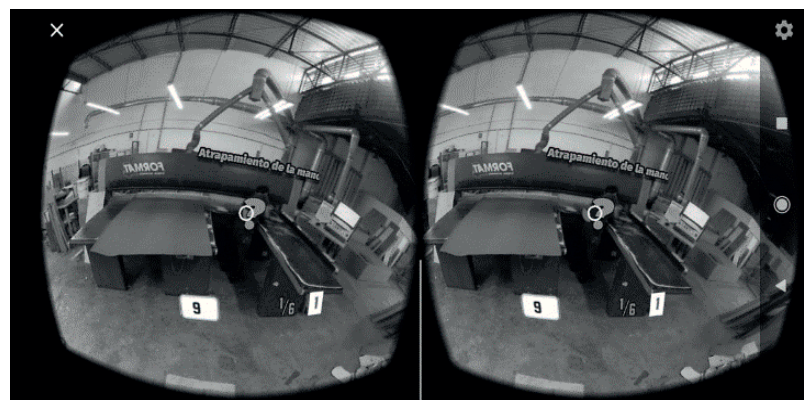

Figura 3. Identificación por parte del usuario de un peligro. Fuente: autores a partir de la aplicación desarrollada. 
Mariana Acuña Rangel, Juan Camilo Lésmez Peralta, Maira Camila Paba Medina, Luis Miguel Salas Chía Desarrollo de una herramienta de aprendizaje experiencial en el área de Seguridad y Salud en el Trabajo soportada en Realidad Virtual

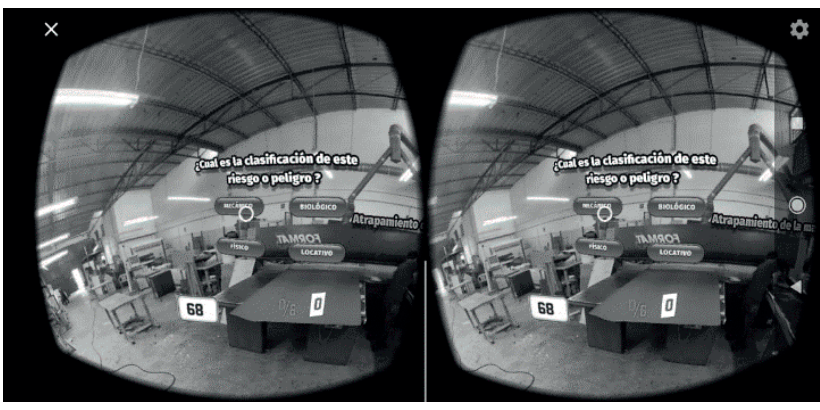

Figura 4. Pregunta al usuario sobre la clasificación del riesgo. Fuente: autores a partir de la aplicación desarrollada.

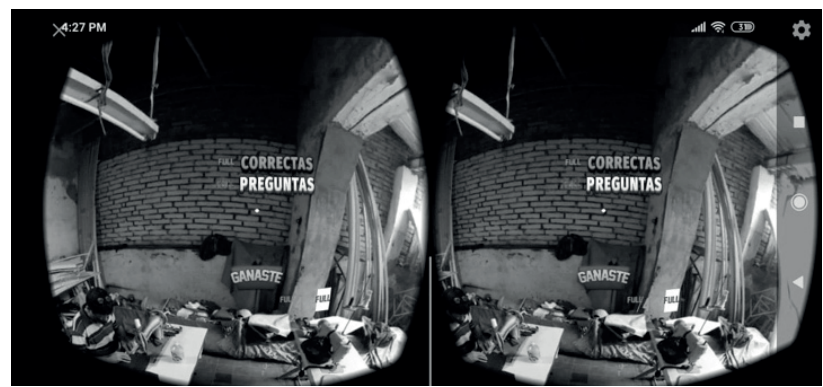

Figura 5. Pantalla al finalizar el escenario identificando y clasificando los tipos de riesgo. Fuente: autores a partir de la aplicación desarrollada.

Como paso final, la validación de la herramienta fue realizada mediante una encuesta que de acuerdo con Lobo-Rueda et al. (2020), es la más utilizada para la evaluación de experiencias educativas. En esta, estudiantes de pregrado que cursaron la asignatura de SST y miembros del Laboratorio de Innovación Educativa GALEA que tuvieron contacto con la herramienta, valoraron aspectos como la facilidad de uso, estética, engagement, enjoyment y utilidad de las preguntas. La encuesta contenía afirmaciones que hacían referencia a cada uno de los aspectos evaluados, a los cuales los participantes debían responder entre una escala Likert que fluctúa de "muy en desacuerdo" a "muy de acuerdo". Los resultados obtenidos fueron de carácter positivo en cada uno de estos aspectos, lo que asegura el correcto funcionamiento de la herramienta.

Para la aplicación de la herramienta se utiliza un celular smartphone con giroscopio en el cual se corre la interfaz y se acompaña de unas gafas de Realidad Virtual que soportan la herramienta y facilitan el componente experiencial para el usuario. Estos dos componentes de hardware son indispensables para la interacción entre los estudiantes y la herramienta de aprendizaje experiencial significativo.

\section{Conclusiones}

A través del análisis de la revisión de literatura, se encuentra que la sociedad actual demanda múltiples capacidades en los jóvenes, necesarias para responder ante los frecuentes cambios en el entorno, entre ellas, se destaca la habilidad para aprender de forma autónoma, efectiva y significativa, lo que impacta en la forma en la que retienen conocimientos por periodos de tiempo más extensos de los habituales (Romero Ariza, 2010). En cuanto al aprendizaje autónomo, este proyecto reafirma lo encontrado por otros investigadores, tales como Paba-Medina et al. (2020) quienes exponen que una forma de alcanzar esta autonomía es haciendo uso de nuevas tecnologías que permitan a los estudiantes tener un mayor abanico de herramientas que faciliten su aprendizaje significativo. Como muestra de ello, los resultados obtenidos en el desarrollo de la presente investigación evidencian la importancia de desarrollar herramientas que promuevan metodologías innovadoras de enseñanza y de aprendizaje, como el aprendizaje experiencial basado en RV, el cual permite una constante interacción entre los usuarios con escenarios ficticios basados en realidad, a los que se deberán enfrentar en situaciones fortuitas o peligrosas, tal como es el caso de los accidentes laborales. Así, se encuentra que esta resulta ser una metodología muy efectiva para aprender de forma práctica y segura.

En cuanto a la metodología, se evidencia la importancia de implementar estrategias que permitan incluir a los usuarios finales en los procesos de diseño de las herramientas didácticas, pues sus necesidades deben ser el principal insumo para la construcción de los objetos de aprendizaje. En este caso, se encuentran insuficiencias en las aulas para la educación práctica en seguridad y salud en el trabajo, en la medida que las problemáticas latentes en esta área surgen en escenarios peligrosos a los que difícilmente se puede enfrentar un estudiante. De esta manera, se reconoce el aprendizaje experiencial basado en RV como una estrategia adecuada para atender a las necesidades de esta población.

Por otro lado, a través del brainstorming se pudo obtener gran cantidad de ideas innovadoras, a partir de las cuales se moldearon los cuatro escenarios incluidos en la herramienta de aprendizaje experiencial significativo enfocada en SST. Para que el brainstorming tuviese mejores resultados fue indispensable realizarlo entre un grupo multidisciplinario, dentro del cual confluyeron diversos saberes que aportaron múltiples ideas relacionadas con las experiencias a las que se enfrentará el estudiante al hacer uso de la RV.

Como punto final, gracias al instrumento diseñado para la validación de la herramienta, se pudo evaluar inicialmente la satisfacción de los usuarios con factores como la facilidad de uso, la estética, el engagement, el enjoyment y la utilidad de las preguntas, midiendo qué tan de acuerdo se encuentran con ciertas afirmaciones que giran en torno a cada factor. Esta validación obtuvo un valor positivo en cada elemento evaluado, presentando el más alto en el denominado enjoyment, recibiendo comentarios por parte de los participantes 
Mariana Acuña Rangel, Juan Camilo Lésmez Peralta, Maira Camila Paba Medina, Luis Miguel Salas Chía

Desarrollo de una herramienta de aprendizaje experiencial en el área de Seguridad y Salud en el Trabajo soportada en Realidad Virtual

que describieron la experiencia en general como "innovadora y práctica", por lo que se asegura que el desarrollo de la herramienta mejora el proceso de aprendizaje sobre identificación y clasificación de riesgos laborales haciéndolo más atractivo para los estudiantes. Además, se espera realizar nuevas validaciones con estudiantes de SST para asegurar con mayor precisión la utilidad de la herramienta.

Esta investigación resalta la importancia de este tipo de herramientas para la educación puesto que aplica metodologías de enseñanza y aprendizaje que aumentan la motivación mejorando la retención de conceptos y fomentando el aprendizaje significativo.

\section{Limitaciones}

Durante la ejecución del proyecto descrito en este artículo, se presentaron algunas limitaciones relacionadas con la emergencia sanitaria ocasionada por la presencia de la COVID-19 en el territorio, que dificultaron el contacto con estudiantes para la validación de la herramienta, así como la captura de los escenarios incluidos dentro de esta.

\section{Agradecimientos}

Agradecemos a la Universidad Industrial de Santander, especialmente a la Convocatoria para el apoyo de iniciativas de innovación didáctica mediante el uso de tecnologías INNOVA-TIC 2020 de la Vicerrectoría Académica y al equipo líder TIC de la Universidad Industrial de Santander por la financiación otorgada para la realización de este proyecto. Adicionalmente, agradecemos a la docente Martha Liliana Torres Barreto, directora del laboratorio de Innovación Educativa GALEA, por el aporte que tanto ella como su equipo de trabajo realizaron en las primeras fases de la metodología.

\section{Referencias}

Álvarez Torres, S. H., y Riaño Casallas, M. I. (2018). La política pública de seguridad y salud en el trabajo: el caso colombiano. Gerencia y Políticas de Salud, 17(35). https://doi.org/10.11144/Javeriana.rgps17-35. ppss

Arias-Flores, H., Jadán-Guerrero, J., y Gómez-Luna, L. (2019). Innovación educativa en el aula mediante Design Thinking y Game Thinking. Hamut'ay, 6(1), 82-95. https://doi.org/10.21503/hamu.v6i1.1576

Bhat, V. D. (2010). Aprendizaje Experiencial. Una Guía para Educadores (1). https://studylib.es/doc/5078995/ aprendizaje-experiencial--una-guía-paraeducadores

Brown, T. (2008). Design Thinking. Harvard Business Review, 86(6), 84.
Burgos García, A. (2010). ¿Cómo integrar la seguridad y salud en educación? Elementos clave para enseñar Prevención en los centros escolares. Profesorado, Revista de Currículum y Formación del Profesorado, 14(2), 273-301.

Castaño Calle, R., González Alonso, F., De castro Hernández, R. M., y Escudero Vidal, J. (2019). Realidad Aumentada y Virtual en el marco de la discapacidad e inclusión desde una perspectiva universitaria. Comunicación y Pedagogía, 317-318, 73-77.

Castaño García, F., y González Morales, N. (2020). Creación de una aplicación móvil hibrida que aplique la metodología del Design Thinking y documente el producto de cada una las fases. [Trabajo de grado, Universitaria Agustiniana]. https:// repositorio.uniagustiniana.edu.co/bitstream/ handle/123456789/1389/GonzalezMoralesNatalia-2020.pdf?sequence $=4$

Castillo-Vergara, M., Alvarez-Marin, A., y Cabana-Villca, R. (2014). Design thinking: how to guide students and business entrepreneurs in the application. Ingeniería Industria, 35(3), 301-311.

Cruz Carbonell, V., Hernández Arias, Á. F., y Silva Arias, A. C. (2020). Cobertura de las TIC en la educación básica rural y urbana en Colombia. Revista Científica Profundidad Construyendo Futuro, 13(13), 39-48. https://doi.org/10.22463/24221783.2578

Dell'Era, C., Magistretti, S., Cautela, C., Verganti, R., y Zurlo, F. (2020). Four kinds of design thinking: From ideating to making, engaging, and criticizing. Creativity and Innovation Management, 29(2), 324-344. https://doi.org/10.1111/caim.12353

Eijkemans, G. (2003). El Programa de Salud Ocupacional de la Oficina Central de la Organización Mundial de la Salud (OMS). En Red Mundial de Salud Ocupacional.

Escartín, E. R. (2000). La Realidad Virtual, una Tecnología Educativa a Nuestro Alcance. Revista de medios y educación, 15, 5-21.

De la Herrán, A. (2011). Técnicas Didácticas Para Una Enseñanza Más Formativa. http://radicaleinclusiva. com/wp-content/uploads/2018/01/teuniv.pdf

Hernández Palma, H., Monterrosa Assia, F., y Muñoz Rojas, D. (2017). Cultura de prevención para la seguridady salud en el trabajo en el ámbito colombiano. Advocatus, 14(28), 35-43. https://doi. org/10.18041/0124-0102/advocatus.28.889 
Mariana Acuña Rangel, Juan Camilo Lésmez Peralta, Maira Camila Paba Medina, Luis Miguel Salas Chía Desarrollo de una herramienta de aprendizaje experiencial en el área de Seguridad y Salud en el Trabajo soportada en Realidad Virtual

IDEO. (2014). Qué es Design Thinking, en qué consiste el proceso. Design Thinking. https://www. designthinking.es/inicio/

Lobo-Rueda, M. A., Paba-Medina, M. C., y Torres-Barreto, M. L. (2020). Análisis descriptivo de experiencias gamificadas para enseñanza y aprendizaje en educación superior en ingeniería. Revista Espacios, 41(16), 21.

López Jiménez, S. F., Lesmes Ortiz, L. V., Álvarez Ochoa, M., Lino Gamiño, J. A., y Reyes Fong, T. (2020). Generación de proyectos de emprendimiento aplicando la metodología Design Thinking. Tecnología Educativa Revista CONAIC, 7(1), 86-91.

Ministerio del Trabajo. (2020). Sistema de Gestión de Seguridad y Salud en el Trabajo. https://www. mintrabajo.gov.co/relaciones-laborales/riesgoslaborales/sistema-de-gestion-de-seguridad-ysalud-en-el-trabajo

Moreno Mendoza, E. F., y Coronel Arenas, M. F. (2018). Hábitos que impiden la adaptación y adopción de la lectura en estudiantes de Administración de Empresas. Revista Científica Profundidad Construyendo Futuro, 9(9), 2-9. https://doi.org/10. $22463 / 24221783.2457$

Organización Internacional del Trabajo. (2011). Sistema de gestión de la SST: una herramienta para la mejora continua (1. ${ }^{a}$ ed.). https://www.ilo.org/safework/in fo/publications/WCMS_154127/lang--es/index.htm

Paba-Medina, M. C., Acosta-Medina, J. K., y TorresBarreto, M. L. (2020). Design of a gamified tool for the development of citizenship competencies. 6th International Conference on Higher Education Advances (HEAd'20). https://doi.org/10.4995/HEAd2 0.2020 .11136

Razzouk, R., y Shute, V. (2012). What Is Design Thinking and Why Is It Important? Review of Educational Research, 82(3), 330-348. https://doi.org/10.3102/00 34654312457429

Romero Ariza, M. (2010). El aprendizaje experiencial y las nuevas demandas formativas. Revista de Antropología Experimental, 10, 89-102.

Smith, M. K. (s.f). David A. Kolb on experiential learning. The encyclopedia of pedagogy and informal education.

Villaescusa Alejo, M. . I. (2019). Mirar el mundo con los ojos del otro. El mapa de empatía. Aula de Secundaria, $34,25-29$. 